\title{
AVALIAÇÃO DE MÉTODOS DE INTERPOLAÇÃO ESPACIAL APLICADOS À PLUVIOSIDADE EM REGIÃO MONTANHOSA NO LITORAL SUL DO ESTADO RIO DE JANEIRO
}

\author{
FARIAS, Orlindo Gomes de - orlindogomes@hotmail.com \\ Universidade Federal Fluminense /UFF \\ FRANCISCO, Cristiane Nunes - cristianenf@id.uff.br \\ Universidade Federal Fluminense /UFF \\ SENNA, Monica Carneiro Alves - monicasenna@id.uff.br \\ Universidade Federal Fluminense /UFF
}

\begin{abstract}
RESUMO: Os estudos da distribuição espacial das chuvas baseiam-se em dados diários de pluviosidade coletados por estações pluviométricas. Aplicando métodos de interpolação, é possível estimar valores pluviométricos para locais sem dados e gerar uma superfície contínua representando a distribuição espacial de chuvas. Existem dois tipos de métodos: determinísticos, baseados na vizinhança para a estimativa de valores ausentes, e estocásticos, que se baseiam na autocorrelação espacial entre valores das amostras. O presente trabalho tem como objetivo avaliar os modelos digitais de distribuição espacial de chuvas, gerados por métodos de interpolação determinístico e estocástico, através da aplicação de técnicas de validação. A área de estudo, região hidrográfica da baía da Ilha Grande, situada no litoral sul fluminense, apresenta uma distribuição espacial da precipitação heterogênea em decorrência, principalmente, da topografia acidentada. Foram aplicados os métodos Curvatura Mínima, Inverso da Distância Ponderada e Krigagem para interpolar dados de dezoito séries de chuva. Os modelos digitais foram validados através do cálculo de medidas de erro e da análise do comportamento espacial das chuvas. Os resultados revelaram a distribuição espacial heterogênea da precipitação na área de estudo nos três modelos gerados, e que método da Krigagem produziu o padrão de distribuição de chuvas que segue a topografia do terreno. Contudo, de acordo com as medidas de erros, o Inverso da Distância Ponderada foi o interpolador que obteve o melhor desempenho. Conclui-se, assim, que o método estocástico, apesar de representar melhor o comportamento espacial, pode não ser adequado na estimativa de dados, quando a variabilidade espacial pluviométrica é alta e o número de estações é insuficiente para geração do semivariograma adequado.
\end{abstract}

Palavras- chaves: distribuição espacial de chuvas, geoestatística, modelos digitais, geoprocessamento

EVALUATION OF SPATIAL INTERPOLATION METHODS APPLIED TO RAINFALL AT A MOUNTAINOUS REGION IN SOUTH COASTAL OF RIO DE JANEIRO STATE

ABSTRACT: The studies of spatial distribution of rainfall are based on precipitation data collected daily by rain gauge. To estimate precipitation values for places without data can be applied the interpolation methods that build continuous surface representing the spatial distribution of rainfall. There are two types of methods: deterministic, based on neighborhood to estimate the lack values, and stochastic, that are based on spatial autocorrelation among the samples. In this way, the present paper aims to evaluate digital models of spatial distribution of rainfall, made by deterministic and stochastic methods, though the application of validation techniques. The study area, Hydrographic Region of Ilha Grande Bay, situated on south coastal of Rio de Janeiro, has a heterogeneous spatial distribution of rainfall due, principally, the rugged topography. It was applied Spline, Inverse Distance Weighted and Krigring methods to interpolate annual rainfall of eighteen rain gauge. The digital models were validated though calculating the error measurement and analyzing of spatial behavior of rainfall. The results revealed that the spatial distribution of rainfall at study area was represented by the three models, and the Krigring method expressed the pattern of spatial distribution 
of rainfall that follows the topography of terrain. However, the Inverse of Distance Weighted got the best performance according to the error measurement. Thus, it can be concluded that stochastic methods may not be adequate to estimate data when the spatial variability of rainfall amount is high and the number of stations is not enough to build an adequate semivariogram.

Keywords: spatial rainfall distribution, geostatistics, digital models, geoprocessing.

\section{INTRODUÇÃO}

Os estudos da distribuição espacial das chuvas baseiam-se nas estações pluviométricas, que medem e registram os dados diários de pluviosidade nos locais onde estão instaladas. Através da interpolação dos dados pluviométricos, é possível estimar valores para locais sem dados e gerar uma superfície contínua com a distribuição espacial de chuvas. A interpolação consiste em um procedimento matemático de ajuste de uma função a pontos não amostrados que se baseia em valores obtidos de pontos observados (LANDIM, 2000), derivando uma superfície contínua que representa a grandeza do evento estudado, denominada como modelo digital ou modelo numérico.

Marcuzzo et al. (2010) ressaltam que, em estudos de precipitação pluviométrica, a interpolação é uma técnica extremamente necessária para áreas com escassez de postos pluviométricos ou regiões com estações irregularmente distribuídas. Segundo Mazzini et al. (2009), a Krigagem pertence ao grupo de interpoladores estocásticos (ou geoestatísticos), enquanto, entre os interpoladores determinísticos, destacam-se o IDP (Inverso da Distância Ponderada) e a Curvatura Mínima (Spline).

Segundo Andriotti (2005) e Landim (2000), o IDP é um modelo determinístico indicado para áreas com alta densidade de pontos (amostras). Baseado na Lei de Tobler, estima as medidas desconhecidas através da média ponderada das medidas conhecidas, dando maior peso a pontos mais próximos. Devido à simplicidade conceitual e facilidade de programação, é um dos métodos de interpolação mais difundidos (LONGLEY et al. 2013). Como é um modelo isotrópico, ou seja, considera peso da distância igual em todas as direções, uma característica típica do IDP é a geração de contornos concêntricos no entorno dos pontos observados.

A Curvatura Mínima também é um modelo determinístico que, por meio de polinômios, gera superfícies suavizadas, independente da distribuição dos dados. Segundo Landim (2000), os contornos gerados são fiéis aos dados amostrados porque estimam os valores máximos e mínimos das amostras conhecidas. De acordo com Andriotti (2005), a superfície interpolada passa por cada uma das amostras disponíveis reproduzindo os valores da variável. Este método é amplamente usado em estudos hidrológicos.

Krigagem, por sua vez, é um algoritmo que utiliza parâmetros geoestatísticos que, através da construção de um semivariograma, infere o comportamento dos dados (esférico, exponencial, gaussiano etc.) (LANDIM, 2000). Este interpolador assemelha-se ao IDP, mas, enquanto este último considera um algoritmo simples baseado na distância, o primeiro leva em consideração a autocorrelação espacial das variáveis regionalizadas calculada pelo semivariograma (SOUZA et al., 2011), consistindo, assim, em um modelo anisotrópico. 
Cada interpolador é eficaz para ser aplicado a determinado evento estudado bem como a características da área de estudo. Desta forma, é importante verificar se o método de interpolação está adequado ao estudo através da comparação dos dados estimados e os observados bem como da análise da distribuição espacial do evento na superfície interpolada (LANDIM, 2000). Para comparar modelos, o cálculo de erros, baseado nas diferenças entre os valores estimados e observados, é um dos procedimentos que deve ser adotado.

Diante do exposto, o objetivo do presente trabalho é avaliar os modelos digitais de distribuição de chuvas, gerados por três métodos de interpolação com dados de estações pluviométricas, através da validação por cálculos de erro e da análise do comportamento espacial das chuvas nas superfícies interpoladas. Por apresentar topografia acidentada e possuir um dos mais altos índices pluviométricos do estado fluminense, a região hidrográfica da baía da Ilha Grande (RHBIG) foi definida como área de estudo.

\section{MATERIAL E MÉTODOS}

\section{ÁREA DE ESTUDO}

A RHBIG, foco do estudo deste trabalho, possui uma área total de 2.195 $\mathrm{km}^{2}$, sendo $1.740 \mathrm{~km}^{2}$ em território fluminense, compreendendo totalmente os municípios de Angra dos Reis e Paraty e, parcialmente, o município de Mangaratiba. No lado paulista, a região hidrográfica abrange os municípios de Arapeí, Bananal, Cunha e São José do Barreiro, (Figura 1).

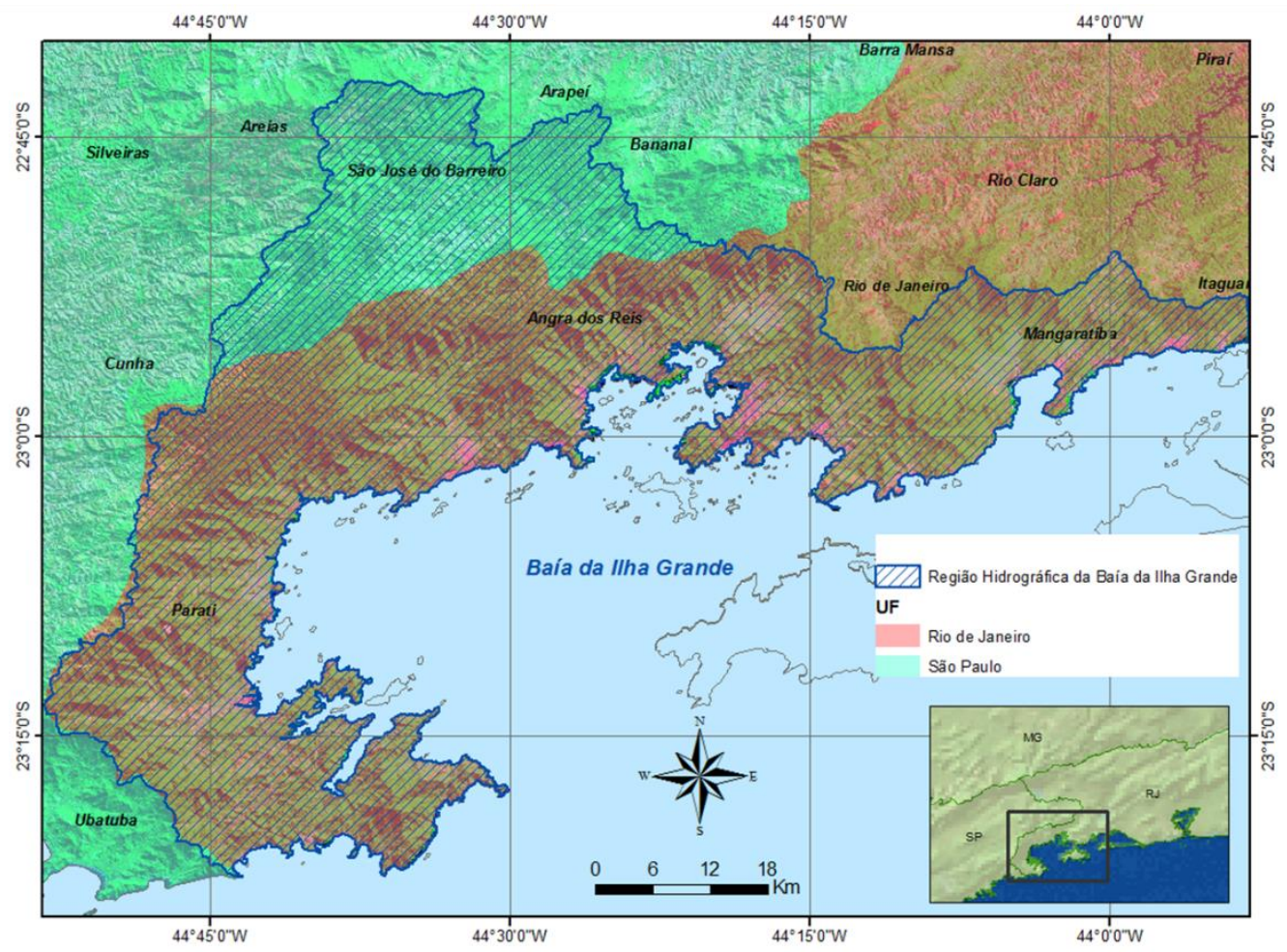

Figura 1 - Região hidrográfica da baía da Ilha Grande (RHBIG), RJ. 
O relevo da faixa continental da RHBIG é caracterizado por escarpas abruptas e por planalto dissecado com vários desníveis, chegando a ultrapassar 2.000 metros de altitude em alguns trechos. A linha de costa apresenta um aspecto recortado, constituída por desembocaduras de cursos d'água de pequeno porte, inúmeras ilhas e enseadas. A região hidrográfica possui uma cobertura vegetal exuberante nas cotas mais elevadas, vegetação de mangue nas enseadas e restinga nas planícies (GUERRA, 2011).

Apresentando um clima Tropical Atlântico, no sul do estado fluminense, onde a Serra do Mar aproxima-se do litoral, bem como na área contínua no litoral paulista, o índice pluviométrico anual ultrapassa $2.500 \mathrm{~mm}$ (DAVIS; NAGHETTINI, 2000). No entanto, a pluviosidade sofre significativa variação ao longo do ano, os seis meses com maior valor pluviométrico na RHBIG, segundo Soares et al. (2014), representam $70 \%$ do valor anual. Conforme Alves et al. (2005), o período de chuvas na RHBIG coincide com o regime pluviométrico da região Sudeste do Brasil, concentrando-se entre os meses de outubro a março, quando ocorrem mais de $80 \%$ do total anual de precipitação. Em decorrência, principalmente, da compartimentação topográfica diversificada, da atuação dos sistemas frontais (SF) originados das altas latitudes e da continentalidade, o Estado do Rio de Janeiro, assim como a região Sudeste, possui uma distribuição espacial da precipitação heterogênea (DERECZYNSKI et al., 2009; NUNES et al., 2009; NIMER, 1979), e elevado índice pluviométrico anual com cerca de $1.500 \mathrm{~mm}$ (REBOITA et al., 2010).

Devido à sua localização geográfica, a RHBIG é a porta de entrada das massas polares no Rio de Janeiro. No sudoeste da Mesorregião Sul Fluminense, onde está inserida a RHBIG, os padrões espaciais e sazonais de chuva são condicionados por sistemas locais (convecção e orografia) a sistemas de brisa marítima, Sistemas de Convecção de Mesoescala (SCM), SF e Zona de Convergência do Atlântico Sul (ZCAS), além da Alta Subtropical do Atlântico Sul (ASAS), que influencia as chuvas da região durante a sua migração sazonal (ANDRÉ et al., 2008; CORREIA et al., 2011). A ZCAS, resultante do encontro das massas de ar oriundas da Amazônia e das latitudes subtropicais, que se estende do noroeste da Amazônia até a costa da Região Sudeste, promove o aumento das chuvas na região. A ASAS, por sua vez, contribui para o aumento da temperatura e diminuição da precipitação (CORREIA et al., 2011).

\section{ETAPAS DA PESQUISA}

Inicialmente foram consultadas e coletadas séries históricas de vinte estações pluviométricas localizadas entre a Serra do Mar e o Planalto da Bocaina, estendendo-se de Mangaratiba (RJ) até Ubatuba (SP). As plataformas utilizadas para a obtenção de dados foram o HIDROWEB (banco de dados hidrológicos da Agência Nacional de Águas - ANA) e o SIGRH (Sistema Integrado de Gerenciamento de Recursos Hídricos - SP), sistema associado ao DAEE (Departamento de Águas e Energia Elétrica - SP).

Das vinte estações pluviométricas selecionadas, oito possuem período de observação entre 25 e 30 anos, o que representa $40 \%$ do total de estações analisadas na área de estudo. Cinco estações possuem período de observação entre 20 e 24 anos, equivalente a $25 \%$ do total de postos pluviométricos, três estações têm período de leitura de dados pluviais entre 15 a 19 anos, ou seja, 
$15 \%$ dos postos, e quatro estações pluviométricas, $20 \%$ das estações, apresentam período de observação inferior a quinze anos (Figura 2).

$\mathrm{Na}$ etapa seguinte, foi realizada a análise de consistência dos dados através do método da média acumulada (dupla massa). Esta técnica identificou erros sistemáticos em algumas estações pluviométricas. Para a análise, as estações pluviométricas foram divididas em dois grupos de acordo com a localização: estações localizadas no litoral e estações situadas no interior.

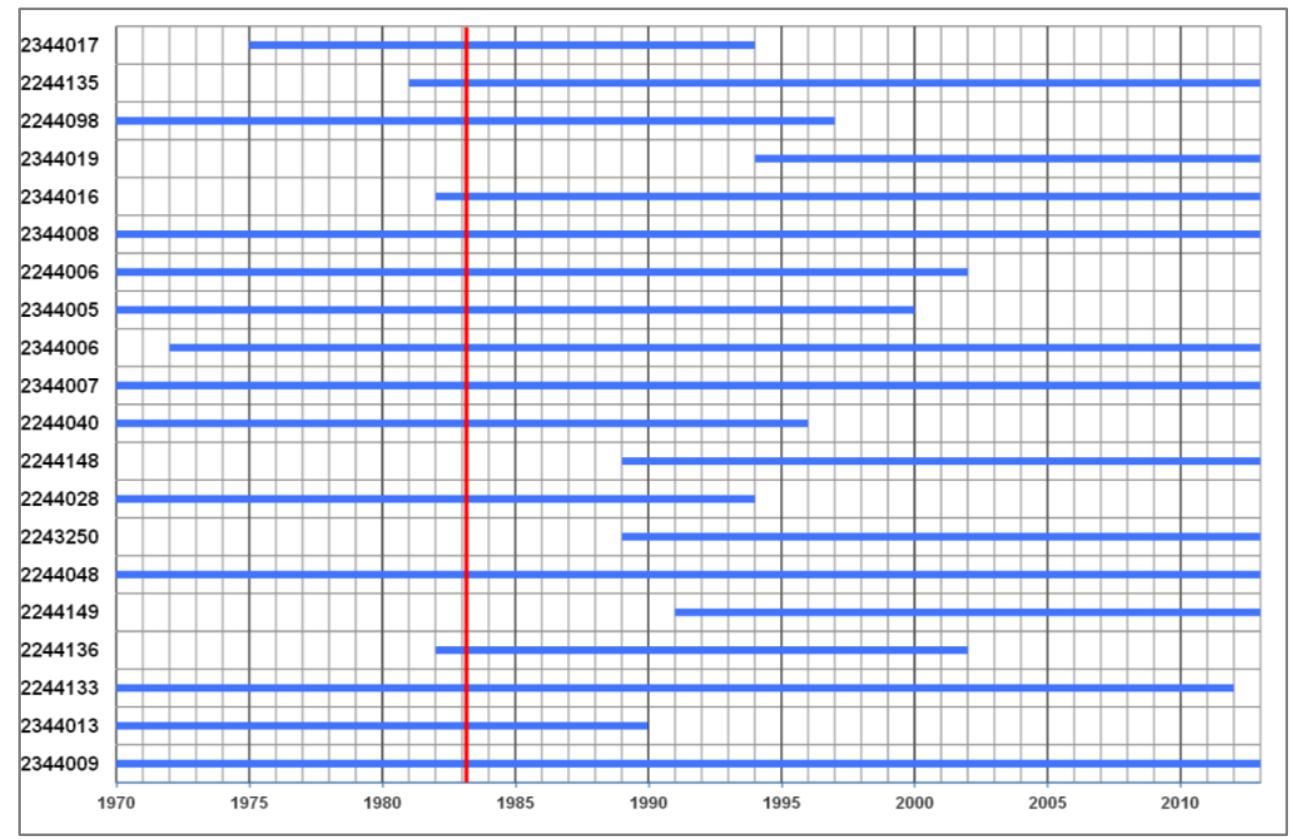

Figura 2 - Estações pluviométricas da RHBIG e do seu entorno.

O método da regressão linear foi utilizado para o preenchimento dos meses sem dados nas estações com falhas nas séries. Para cada série pluviométrica falhada, foram avaliadas as estações que apresentavam maior proximidade geográfica, série histórica com menor número de falhas e longo período de operação.

Em seguida, as séries pluviométricas foram georreferenciadas e classificadas de acordo com o tamanho da série histórica de dados disponíveis, considerando o período de 1983 até 2012. Como nem todas as séries apresentavam período de dados em comum, o total anual de chuvas foi calculado para dezoito estações selecionadas. Com base nestes valores, foram gerados os modelos digitais de distribuição espacial das chuvas com a utilização dos interpoladores Curvatura Mínima com Tensão, IDP e Krigagem, disponíveis na extensão 3D Analyst do ArcGIS 10.

O interpolador Curvatura Mínima utiliza polinômios para ajustar a superfície a ser estimada apresentar uma curvatura mínima passando por todas as amostras. Os cálculos de derivação são efetuados repetidamente até que a diferença (convergência ou tolerância) seja alcançada entre os valores amostrados e os estimados, ou até que um número máximo de interações seja 
alcançado (LANDIM, 2000). Esta técnica consiste, assim, em dividir o intervalo de interesse em intervalos menores e interpolar com polinômios de grau pequeno de modo a criar uma superfície mais suave possível. A opção Curvatura Mínima com Tensão foi utilizada neste trabalho pois controla a rigidez da superfície, criando uma menos suavizada e com valores próximos ao intervalo dos dados amostrados (ESRI, 2010).

No interpolador IDP, o ponto a ser estimado é calculado pela média ponderada das amostras vizinhas cujos pesos são definidos pelo inverso da distância entre as amostras e o ponto a ser estimado (LANDIM, 2000). Para que as amostras mais próximas exerçam maior influência, minimizando as mais distantes, deve ser atribuído maior valor ao expoente dos pesos; caso o objetivo seja distribuir de forma equitativa a sua influência, independente da distância, utiliza-se expoente igual ou menor que dois (FERREIRA, 2014). No presente estudo, foi adotado o valor quatro como potência do peso de modo que as estações mais distantes tivessem menos influência na estimativa dos valores desconhecidos.

A Krigagem também é um interpolador baseado na distância, no entanto a sua principal diferença com os interpoladores determinísticos, como o IDP, é detecção da variabilidade espacial dos dados através da construção de semivariogramas experimentais, expressos em uma representação gráfica com a variância entre as amostras (eixo y) e a distância entre elas (eixo x). Como são experimentais, é necessário ajustá-los a um modelo teórico definido a partir de equações matemáticas sobre as quais os valores dos pontos desconhecidos são estimados (YAMAMOTO, LANDIM, 2013; FERREIRA, 2013).

Por fim, foi realizada a validação dos modelos gerados pelos métodos de interpolação. Devido ao número escasso de postos, foi necessário retirar uma estação do conjunto de dados, realizar nova interpolação e, a seguir, cotejar o valor estimado sem a estação com o valor observado na respectiva estação. Das dezoito estações utilizadas na interpolação, nove foram utilizadas na validação, por se localizarem na área de estudo. Os respectivos procedimentos foram repetidos com os três algoritmos de interpolação, resultando na confecção de 27 modelos de distribuição de chuvas. Foram calculadas as seguintes medidas de erros:

- $\quad$ Soma da diferença (di): diferença entre o valor estimado (Pie) e o valor observado (Pio) (Equação 1). De acordo com Hallack e Pereira-Filho (2011), di $=0$ indica simulação perfeita, no entanto di $>0$ ou di $<0$ indicam simulação imperfeita, sendo que, quanto mais distante de 0 o valor de di, mais imperfeita a simulação.

$d i=P_{i e}-P_{i o}$

(Equação 1)

- $\quad$ Erro Médio (EM): somatório de di dividido pelo número observações $(N)$ (Equação 2). O EM, também chamado de erro sistemático, mede a tendência do modelo em superestimar ou subestimar os valores observados (HALLACK; PEREIRA-FILHO, 2011).

$$
E M=\frac{1}{N} \sum_{i=1}^{N} d i
$$


- $\quad$ Erro Médio Absoluto (EMA): somatório absoluto di dividido pelo número de observações (Equação 3). O EMA, ao considerar os valores absolutos, contorna a possível subestimação dos erros causada pelo EM, que utiliza erros individuais positivos e negativos de mesma magnitude (HALLACK; PEREIRAFILHO, 2011).

$E M A=\frac{1}{N} \sum_{i=1}^{N}\left(\left|P_{i e}\right|-\left|P_{i o}\right|\right)$

(Equação 3)

- $\quad$ Erro Quadrático Médio (EQM): somatório das diferenças individuais ao quadrado dividido pelo número de observações (Equação 4). O EQM é sensível aos grandes erros por elevar as diferenças individuais ao quadrado (HALLACK; PEREIRA-FILHO, 2011).

$E Q M=\frac{1}{N} \sum_{i=1}^{N}\left(P_{i e}-P_{i o}\right)^{2}$

(Equação 4)

- $\quad$ Raiz do Erro Quadrático Médio (REQM): raiz quadrada do somatório das diferenças individuais ao quadrado dividido pelo número de observações (Equação 5). Ao extrair a raiz quadrada, a REQM apresenta valores do erro nas mesmas dimensões da variável analisada, sendo comumente usada para expressar a acurácia dos modelos numéricos (HALLACK; PEREIRA-FILHO, 2011).

$R E Q M=\sqrt{\frac{1}{N} \sum_{i=1}^{N}\left(P_{i e}-P_{i o}\right)^{2}}$

(Equação 5)

\section{RESULTADOS E DISCUSSÃO}

A análise dos modelos de distribuição de chuvas gerados pelos métodos Curvatura Mínima, IDP e Krigagem demonstrou um comportamento heterogêneo na distribuição espacial da precipitação na RHBIG. As chuvas concentram-se no litoral e na escarpa da Serra do Mar, correspondendo às estações pluviométricas situadas a barlavento, com valores alcançando até $2.600 \mathrm{~mm}$, enquanto tendem a diminuir no interior da faixa continental da RHBIG, no reverso da Serra do Mar e no Planalto da Bocaina, com valores atingindo $1.300 \mathrm{~mm}$.

As estações Ibicuí e Parati se revelaram como anômalas em relação aos demais postos pluviométricos situados no litoral, apresentando volume de chuvas comparável às estações do interior. A presença da Ilha Grande, localizada a sudoeste da estação de Ibicuí, pode explicar o menor volume de chuvas nesta estação, bem como a localização da estação Paraty, que fica rodeada por barreiras topográficas. Por outro lado, as estações que apresentam os maiores totais anuais de precipitação foram Patrimônio, Picinguaba, São Roque e Bracuí, todas situadas no litoral, onde não há a presença de uma barreira topográfica a barlavento. 
O método Curvatura Mínima produziu uma superfície suavizada, sendo, assim, a influência das estações com valores extremos tendeu a se apresentar de modo atenuado no modelo se comparado com os outros dois interpoladores aqui analisados (Figura 3a). Por lado, como este interpolador não altera os valores dos dados observados, a superfície gerada apresenta amplitudes bruscas próximas, como nas proximidades das estações Fazenda Fortaleza e Vila Mambucaba. Segundo Landim (2000), a Curvatura Mínima não é apropriada para mudanças bruscas do terreno em curtas distâncias, pois pode exagerar nos valores dos pontos interpolados.

O modelo gerado pelo método IDP apresentou contornos concêntricos, em formato de vórtices, ao redor das estações, principalmente daquelas que apresentam valores extremos, como Paraty, São Roque, Patrimônio, Bracuí, Bananal e Campos do Cunha (Figura 3b). As feições concêntricas representam a ponderação da distância no entorno das estações. De acordo com Landim (2000), o IDP é um algoritmo que destaca as variações de pequena amplitude (irregularidades locais) em regiões com dados dispersamente distribuídos.

O modelo gerado pela Krigagem apresentou uma superfície suavizada e sem a presença de vórtices ao redor das estações (Figura $3 c$ ), pois os valores são calculados com base na autocorrelação espacial das variáveis regionalizadas. Também os valores dos pontos observados são alterados no modelo, diferindo da Curvatura Mínima, que mantém os respectivos valores e cria mudanças bruscas no terreno. Verificou-se, pela análise do comportamento espacial da distribuição de chuva, que a superfície gerada pela Krigagem acompanhou a topografia, com volume pluviométrico diminuindo para o interior e seguindo padrão paralelo à direção da escarpa da Serra do Mar até atingir os divisores d'água. 

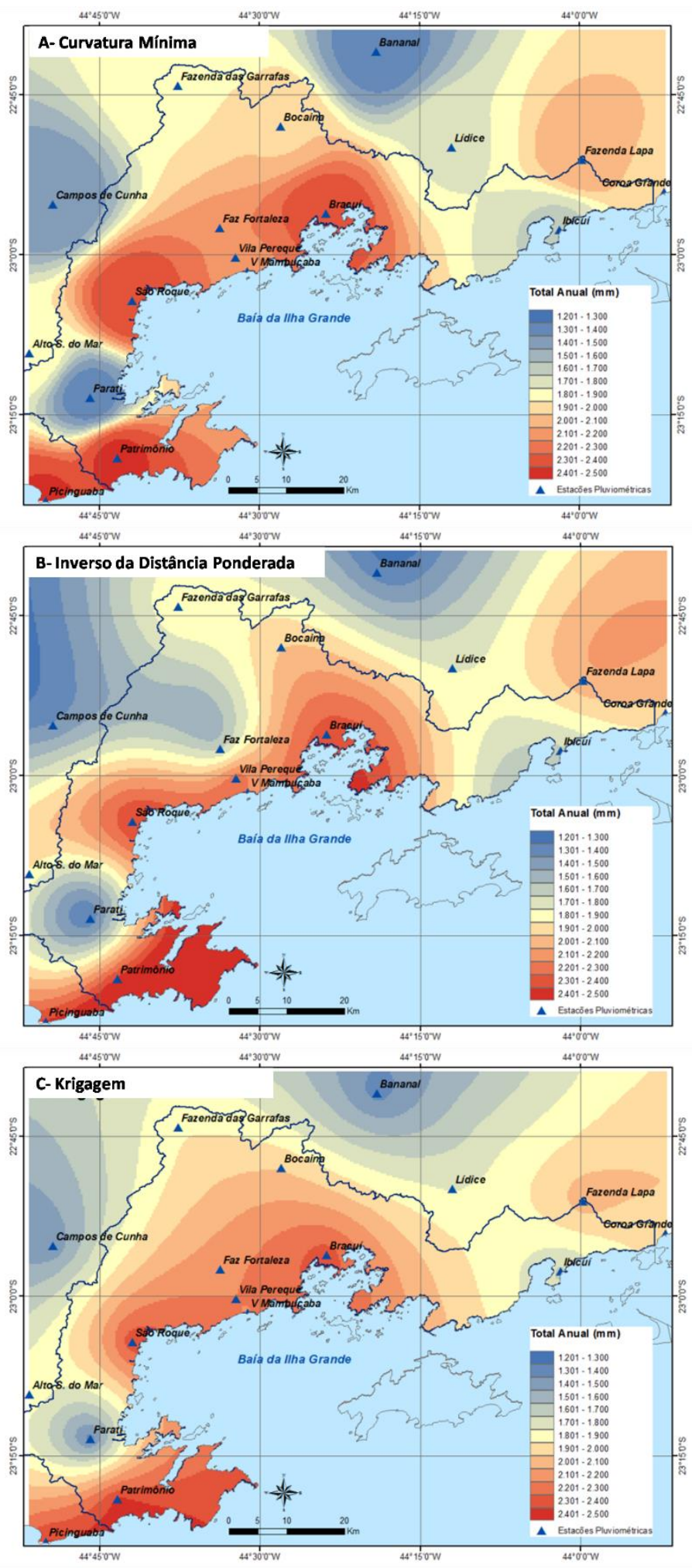

Figura 3 - Distribuição espacial das chuvas na RHBIG e no entorno gerada pelo método (a) Curvatura Mínima, (b) IDP e (c) Krigagem. 
O semivariograma, gerado com as 18 séries pluviométricas, foi ajustado ao modelo exponencial, pois apresentou o menor erro padrão médio entre os valores observados e os estimados, $365 \mathrm{~mm}$, em comparação aos demais modelos disponibilizados pelo algoritmo (esférico, circular, gaussiano e linear). O modelo exponencial é considerado moderadamente não estacionário, ou seja, a medida que a distância entre as amostras aumenta, a variância tende a apresentar valores menores (Figura 4).

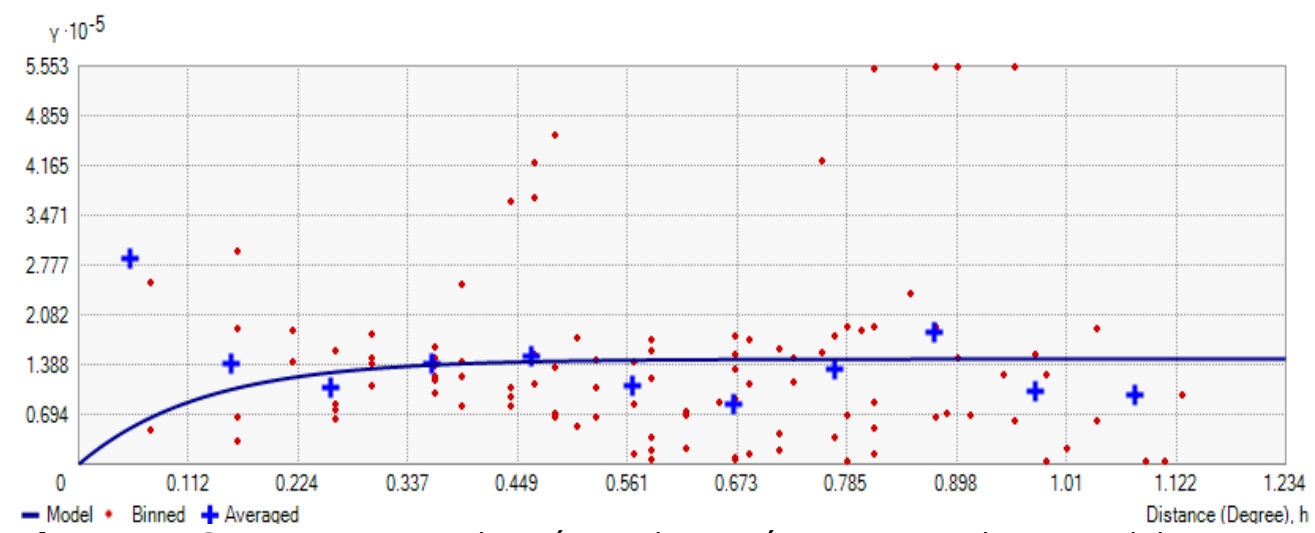

Figura 4 - Semivariograma das séries pluviométricas ajustado ao modelo exponencial.

No entanto, pela validação das medidas de erro, verifica-se que o interpolador IDP obteve os menores valores de erros médios (Tabela 1). O EM indica que houve subestimação próxima a $50 \mathrm{~mm}$ do valor calculado o pelo método Krigagem, enquanto para IDP e Curvatura Mínima o erro foi próximo a zero. No entanto, o EMA demonstra que os três interpoladores ficaram com erros acima de $300 \mathrm{~mm}$ e, assim, destaca que o menor valor do EM alcançado pelo IDP ocorreu pela compensação entre valores individuais superestimados e subestimados. Em geral, as medidas de erro demonstram que a Curvatura Mínima apresentou pior desempenho, seguido da Krigagem, principalmente, os erros quadráticos que acentuam as diferenças individuais.

Apenas três estações - Fazenda das Garrafas, Lídice e Bocaina, localizadas no interior da RHBIG e com total pluviométrico anual entre 1.800 a $2.000 \mathrm{~mm}$, apresentaram a maior parte de di inferior a $100 \mathrm{~mm}$ (Tabelas $1 \mathrm{e}$ 2). As outras seis estações apresentaram a maioria das di acima de $300 \mathrm{~mm}$ e possuem valores de chuva extremos. As estações de Ibicuí, Vila Mambucaba e Parati, com total pluviométrico anual entre 1.400 a $2.200 \mathrm{~mm}$, apresentaram valores superestimados, com diferença variando entre 300 a $1.000 \mathrm{~mm}$, pois, apesar de localizarem no litoral, não seguem o padrão das estações vizinhas de chuvas com índices elevados. Enquanto as estações de Bracuí, São Roque e Patrimônio, situadas próximo ao litoral, com total pluviométrico anual próximo de $2.400 \mathrm{~mm}$, apresentaram valores subestimados, com di alcançando até 800 $\mathrm{mm}$.

Apesar dos valores das di serem acentuadas em 2/3 das estações testadas, a média do total pluviométrico anual dos valores observados e estimados pelos três interpoladores é muito próxima, em torno de $2.000 \mathrm{~mm}$. No entanto, o desvio-padrão dos valores observados é cerca de $360 \mathrm{~mm}$, semelhante ao valor estimado pela Curvatura Mínima e superior aos valores estimados pelos outros dois interpoladores. 
Este trabalho chegou a resultados semelhantes a Magalhães et al. (2013) que executaram a espacialização da precipitação pluvial na região norte do estado do Espírito Santo, com aplicação dos métodos IDP, Tendência, Krigagem e Curvatura Mínima. Após interpolar 54 séries e validar com 49 séries pluviométricas, os autores concluíram que IDP foi o mais eficiente para a distribuição espacial da chuva por apresentar menor valor de erro e índice de concordância mais próximo de um. No entanto, Deus et al. (2010), em trabalho realizado com séries de 28 postos pluviométricos na bacia do alto e médio Rio Teles Pires, no estado de Mato Grosso, afirmam que o algoritmo Krigagem obteve o melhor desempenho, na espacialização de chuvas, nos indicadores Tendência (Bias), Raiz do Erro Médio Quadrático e Razão da Variância.

Tabela 1 - Medidas de erros entre os valores de chuva observados e estimados pelos interpoladores.

\begin{tabular}{crrr}
\hline \multirow{2}{*}{ Estação Pluviométrica } & \multicolumn{3}{c}{ Diferença $\left(\mathrm{d}_{\mathrm{i}}\right)$} \\
\cline { 2 - 4 } & $\begin{array}{c}\text { Curvatura } \\
\text { Mínima }\end{array}$ & Krigagem & \multicolumn{1}{c}{ IDP } \\
\hline Faz. Garrafas & -61 & -76 & 8 \\
Bocaina & -8 & -54 & -1 \\
Lídice & 1 & 55 & 152 \\
Ibicuí & 527 & 311 & 324 \\
V. Mambucaba & 377 & 220 & -80 \\
Parati & 929 & 947 & 875 \\
Bracuí & -269 & -375 & -367 \\
São Roque & -771 & -824 & -447 \\
Patrimônio & -732 & -686 & -462 \\
\hline EM & -1 & -53 & 0,24 \\
EMA & 409 & 394 & 302 \\
EQM & 276.844 & 260.726 & 161.208 \\
REQM & 526 & 510 & 401 \\
\hline
\end{tabular}

Tabela 2 - Total pluviométrico anual observado e estimado pelos interpoladores. 


\begin{tabular}{ccccc}
\hline \multirow{2}{*}{ Estação } & \multicolumn{4}{c}{ Precipitação Pluviométrica (mm) } \\
\cline { 2 - 5 } & Observado & $\begin{array}{c}\text { Curvatura } \\
\text { Mínima }\end{array}$ & Krigagem & IDP \\
\hline Faz. Garrafas & 1.887 & 1.825 & 1.810 & 1.895 \\
Bocaina & 1.992 & 1.984 & 1.938 & 1.990 \\
Lídice & 1.792 & 1.794 & 1.848 & 1.945 \\
Ibicuí & 1.682 & 2.209 & 1.993 & 2.006 \\
V. Mambucaba & 2.195 & 2.573 & 2.415 & 2.114 \\
Parati & 1.351 & 2.280 & 2.298 & 2.227 \\
Bracuí & 2.351 & 2.082 & 1.975 & 1.984 \\
São Roque & 2.376 & 1.604 & 1.552 & 1.929 \\
Patrimônio & 2.419 & 1.686 & 1.733 & 1.957 \\
\hline Média & 2.005 & 2.004 & 1.952 & 2.006 \\
Desvio Padrão & 363 & 313 & 268 & 103 \\
\hline
\end{tabular}

\section{CONSIDERAÇÕES FINAIS}

O presente trabalho avaliou, através da análise do comportamento espacial da chuva e medidas de erro, três modelos digitais de distribuição pluviométrica, produzidos com base em séries históricas de estações pluviométricas, localizadas na RHBIG e no seu entorno, utilizando diferentes métodos de interpolação.

De acordo com medidas de erro, conclui-se que o IDP apresentou o melhor desempenho entre os interpoladores testados - Krigagem e Curvatura Mínima, pois obteve os menores valores tanto de erros médios quanto das diferenças individuais entre os valores observados e estimados. Também foi este interpolador que apresentou menor erro médio em relação à superestimação e subestimação dos valores estimados.

Verificou-se, também, que as maiores medidas de erros foram obtidas por estações pluviométricas com valores extremos. As estações situadas no litoral, com total anual pluviométrico elevado, foram subestimadas, no entanto, aquelas com total menor, também situadas no litoral, apresentaram valores superestimados.

Por outro lado, as estações pluviométricas que apresentaram a menores diferenças entre os dados observados e estimados estão mais afastadas do litoral, próximas ao divisor de águas da RHBIG.

No entanto, ao analisar o comportamento espacial de chuvas dos modelos gerados pelos três interpoladores, verifica-se que método da Krigagem produziu um resultado plausível, pois o padrão de distribuição de chuvas segue a topografia do terreno conforme indicado pelos dados observados das estações pluviométricos da RHBIG.

Apesar das incertezas dos resultados aqui alcançados, devido ao número reduzido de estações pluviométricas existentes na área de estudo, pode-se afirmar que o desempenho atingido pelos interpoladores foi influenciado pela heterogeneidade da distribuição espacial de chuvas na RHBIG. Pois, caso não haja um número de estações que contemple o comportamento espacial, o modelo tende a não apresentar a variabilidade 
existente. Posto isto, o baixo desempenho alcançado pela Krigagem, indicado pelas medidas de erro, pode estar relacionado à alta variabilidade espacial do volume pluviométrico na RHBIG, em função de topografia acentuada do terreno, ou seja, o método geoestatístico pode não se mostrar adequado para a modelagem de mudanças bruscas, quando o número de pontos observados é insuficiente para geração do semivariograma que represente a variabilidade espacial.

\section{REFERÊNCIAS BIBLIOGRÁFICAS}

ALVES, L. M.; MARENGO, J. A.; CAMARGO JR., H.; CASTRO, C. Início da estação chuvosa na região Sudeste do Brasil: Parte 1 - Estudos observacionais. Revista Brasileira de Meteorologia, v.20, n.3, p 385-394. 2005.

ANDRÉ, R. G. B.; MARQUES, V. S., PINHEIRO, F. M. A., FERRAUDO, A. S. Identificação de regiões pluviometricamente homogêneas no estado do Rio de Janeiro, utilizando-se valores mensais. Revista Brasileira de Meteorologia, v.23, n. 4, p. 501-509, 2008.

CORREIA, T. P.; COSTA, C. D.; OLIVEIRA-JÚNIOR, J. F.;MORAES, N. O.; LYRA, G. B. Distribuição espacial da precipitação pluvial mensal no Estado do Rio de Janeiro. In: Congresso Brasileiro de Agrometeorologia, 17., 2011, Guarapari. Anais... Guarapari, ES: SBAgro. 2011.6 p.

DAVIS, E. G.; NAGHETTINI, M. C. Estudo de chuvas intensas no Estado do Rio de Janeiro. Brasília: CPRM, 2000.

DEUS, B. V.; ZEILHOFER, P.; ARAUJO, G. C.; SANTOS, A. S. L. dos. Interpolação Pluviométrica na Bacia do Alto e Médio Rio Teles Pires: uma análise de séries históricas e interpoladores. In: Simpósio Brasileiro de Ciências Geodésicas e Tecnologias da Geoinformação, 3., 2010, Recife. Anais... Recife: CTG/UFPE. 2010. p 1-7.

DERECZYNSKI, C. P.; OLIVEIRA, J. S.; MACHADO, C. O. Climatologia da precipitação no município do Rio de Janeiro. Revista Brasileira de Meteorologia, v.24, n.1, p 24-38, 2009.

ESRI. Interpolating Surfaces in ArcGIS Spatial Analyst. ArcUser, JulhoSetembro, 2004. Disponível em: http:// www.esri.com/news/arcuser/0704/fles/interpolating.pdf. Acesso em: 07 abril 2013

FERREIRA,M.C. Iniciação à Análise Geoespacial: teoria, técnicas e exemplos em Geoprocessamento. São Paulo: Editora UNESP, 2014. 343 p.

HALLACK, R.; PEREIRA-FILHO, A.J. Metodologia para análise de desempenho de simulações de sistemas convectivos na região metropolitana de São Paulo com o modelo ARPS: sensibilidade a variações com os esquemas de advecção e assimilação de dados. Revista Brasileira de Meteorologia, v.26, n.4, p 591608, 2011.

LANDIM, P.M.B. Introdução aos métodos de estimação espacial para confecção de mapas. Rio Claro: UNESP, 2000. 20 p.

LONGLEY, P.A.; GOODCHILD, M.F.; MAGUIRE, D.J.; RHIND, D.W. Sistemas e ciência da informação geográfica. Porto Alegre: Bookman, 2013. 540p. 
MAGALHÃES, I. .A. L.; ALMEIDA, K. L.; THIAGO, C. R. L.; JUNIOR, B. S. G.; ZANETTI, S. S.; CECÍlIO, R. A.; Análise de métodos de interpolação da precipitação pluvial na região norte do estado do Espírito Santo, Brasil. In: Simpósio Brasileiro de Sensoriamento Remoto, 16., 2013, Foz do Iguaçu. Anais... Foz do Iguaçu:INPE, 2013. p. 5651-5657.

MARCUZZO, F. F. N.; CARDOSO, M. R. D.; MELLO, L. T. A. Uso dos Métodos de Krigagem e Spline de Tensão no Mapeamento de Chuvas na Região Metropolitana de Goiânia e Seu Entorno. In: Simpósio Internacional Caminhos Atuais da Cartografia na Geografia, 2., 2010, São Paulo. Anais... São Paulo: EDUSP, 2010.

MAZZINI, P. L. F.; SCHETTINI, C. A. F. Avaliação de metodologias de interpolação espacial aplicadas a dados espaciais costeiros quase-sinóticos. Braz J. Aquat. Sci. Technol. v. 13, n.1, p 53-64, 2009.

NIMER, E. Climatologia do Brasil. Rio de Janeiro: IBGE, 1979, 422 p.

NUNES, L. H.; VICENTE, A. K.; CANDIDO, D. H. Clima da região sudeste do Brasil. In: CAVALCANTI, I. F. A.; FERREIRA, N. J.; JUSTI DA, M. G. A.; SILVA DIAS, M. A. F. Tempo e clima no Brasil. São Paulo: Oficina de textos, 2009. Cap. 16, p. 243-256.

REBOITA, M. S., GAN, M. A., DA ROCHA, R. P., AMBRIZZI, T. Regimes de Precipitação na América do Sul: Uma Revisão Bibliográfica. Revista Brasileira de Meteorologia, v. 25, n. 2, p 185-204. 2010.

SOARES, F. S.; FRANCISCO, C. N.; SENNA, M. C. A. Distribuição EspaçoTemporal da Precipitação na Região Hidrográfica da Baía da Ilha Grande - RJ. Revista Brasileira de Meteorologia, v.29, n.1, p 125-138. 2014.

SOUZA, J. L. L. L; GOMES, T. S.; DIAS, R.S.; OLIVEIRA, G. M. A.; SANTOS, R. L. Avaliação de métodos de interpolação aplicados à espacialização das chuvas no território identidade Portal do Sertão / Bahia. In: Simpósio Brasileiro de Sensoriamento Remoto, 15., 2011, Curitiba. Anais... São José dos Campos: INPE, p 4295-4302. 2011

YAMAMOTO, J.K.; LANDIM, P.M.B. Geoestatística: Conceitos e Aplicações. São Paulo: Oficina dos Textos, 2013. 215p. 